\title{
A formula to calculate the contrast volume required for optimal imaging quality in optical coherence tomography with non-occlusive technique
}

\author{
Juan Luis Gutiérrez-Chico ${ }^{1,2}$, Carlos Cortés ${ }^{1,3}$, \\ Michele Schincariol ${ }^{2}$, Milosz Jaguszewski ${ }^{4}$ \\ ${ }^{1}$ DRK Klinikum Westend, Berlin, Germany \\ ${ }^{2}$ Institute of Cardiovascular Translational Research of Atlantic (ICTRA), Berlin, Germany \\ ${ }^{3}$ Hospital Clínico Universitario de Valladolid, ICICOR, Spain \\ ${ }^{4}$ First Department of Cardiology, Medical University of Gdańsk, Poland
}

This paper was guest edited by Prof. Francesco Prati

\begin{abstract}
Background: Non-occlusive technique is universally accepted for acquisition of coronary optical coherence tomography (OCT), but the amount of contrast infused is still inconsistently calculated. Proposed herein, is an empirical formula for accurate contrast volume calculation.

Methods: In an observational prospective study, contrast volume of consecutive patients undergoing OCT was either calculated with formula, or eyeballed based on manufacturer recommendations. The quality of pullback, defined as \% of high quality cross-sections (CS) in the segment of interest (SOI), was analyzed by two independent operators and compared between groups, together with the amount of contrast per pullback.

Results: Sixty patients (115 pullbacks, 4252 CS) were imaged using the formula, vs. 18 patients (22 pullbacks, 777 CS) eyeballing the contrast volume. The formula group used $18 \mathrm{~mm} / \mathrm{s}$ as pullback speed more often (82.6\% vs. $40.9 \%, p=0.0001)$, but there were no significant differences between groups in SOI length or vessel imaged. The formula resulted in higher pullback quality than eyeballing (96.55\% vs. 63.55\%, $p<0.0001)$, interobserver agreement Kappa $0.903(p<0.0001)$, and tended to use less contrast per pullback than the eyeball group (13.03 $\mathrm{mL}$ vs. $14.55 \mathrm{~mL}, p=0.057)$. After adjusting for pullback speed, SOI length and vessel in multivariate linear regression, the use of the formula significantly reduced the amount of contrast in $4.50 \mathrm{~mL}$ on average.

Conclusions: Optical coherence tomography acquisition with the non-occlusive technique can be substantially eased with the use of a novel formula to calculate the contrast volume required. This method optimises the quality of the images whilst reducing the amount of contrast per pullback. (Cardiol J 2018; 25, 5: 574-581)
\end{abstract}

Key words: tomography, optical coherence, coronary heart disease

Editorial p. 569

\section{Introduction}

Optical coherence tomography (OCT) is increasingly gaining acceptance in interventional cardiology and it currently disputes the position as preeminent intracoronary imaging modality to intravascular ultrasound (IVUS) at eye level [1]. Although OCT was initially perceived as rather a research tool, its potential for problem-solving in complex coronary interventions is huge and guidelines have recently recommended the use

Address for correspondence: Prof. Juan Luis Gutiérrez-Chico, MD, PhD, FESC, FACC, Head of Interventional Cardiology, DRK Klinikum Westend, Spandauer Damm 130, 14050 - Berlin, Germany, tel: +49 (0) 176 30585019, +34 615 319370 , e-mail: juanluis.gutierrezchico@ictra.es

Received: 26.12.2017 Accepted: 11.09.2018 
of OCT in routine invasive procedures for several indications [2]. All OCT systems from different manufacturers currently implemented for clinical applications rely on Fourier-domain interferometry, which allows much faster acquisition of massive amounts of information. This has enabled the development of the non-occlusive acquisition technique, which makes the most of translucency and viscosity of contrast media: viscosity removes the blood completely from the arteries during coronary angiography, whereas translucency permits recording the whole OCT pullback during the brief instant in which the arteries are filled with contrast $[3,4]$. OCT non-occlusive acquisition technique is at present universally used in clinical applications. It requires infusion of a certain contrast volume at a continuous infusion rate $(3-4 \mathrm{~mL} / \mathrm{s})$, so the most refined OCT experts use an automatic injector in order to warrant a minimal quality of acquired images [1]. Whilst there is consensus regarding recommended infusion rate for an optimal OCT acquisition, it is less clear how much contrast must be infused by automatic injector. Adjusting the volume of contrast to a minimum is mandatory to prevent the risk of nephropathy, but the quality of OCT also depends on a sufficient amount of contrast, so the whole segment of interest (SOI) can be imaged without shadowing or artefacts derived from incomplete flushing. A sober balance is hence essential for an accurate calculation of contrast volume required for each OCT acquisition. This calculation becomes even more challenging, since different pullback speeds and lengths are currently available in modern OCT systems.

A simple formula to calculate minimal contrast volume required in non-occlusive technique for optimal OCT acquisition is hereby proposed, and compared vs. standard approximate estimations in terms of image quality and total amount of contrast used.

\section{Methods}

This is an observational prospective singlecenter study comparing a simple formula for accurate calculation of the contrast volume vs. standard approximate estimations for OCT acquisition with the non-occlusive technique, aiming at two different variables: image quality and contrast volume.

\section{Study population}

Patients undergoing OCT at the Hospital of Frankfurt Oder (Germany) between 10/01/2016 and $31 / 12 / 2016$, irrespective of clinical indication, were included in the study. Exclusion criteria were: 1) Inability to engage coronary ostium properly with guiding catheter, preventing an adequate coronary flushing; 2) Occlusive OCT catheter; 3) Non-uniform rotational distortion (NURD) artefact; 4) Operators acquiring OCT imaging with manual injection of contrast.

The study was conducted according to the Declaration of Helsinki. Since all steps, hereby described in the intervention, conform to currently approved standards of good clinical practice and only data stemming from a single centre were analyzed, the approval of the Ethics Committee could be waived and no specific informed consent was provided to patients, other than the ones conventionally used for diagnostic coronary angiography, percutaneous coronary intervention and for the use of invasive imaging.

\section{OCT acquisition}

The diagnostic or therapeutic procedure was performed via radial or femoral access with $\mathrm{a} \geq 6 \mathrm{~F}$ guiding catheter without side holes. A conventional 0.014" PCI wire at the operator's choice was advanced distally to the target segment. The length of SOI was estimated angiographically using customary references in the wire or guiding catheter, including sufficient proximal and distal margins to ensure imaging of the whole target segment with optimal quality. OCT pre-intervention was acquired with a Dragonfly catheter and an Ilumien Optis console (St. Jude Medical, St. Paul, MN, USA) at a rotation speed of $180 \mathrm{~Hz}$ and a pullback speed of 18 or $36 \mathrm{~mm} / \mathrm{s}$, at the operator's discretion, according to a non-occlusive technique [3], using an automatic power injector (Medrad Avan$\mathrm{ta}^{\mathrm{TM}}$, Medrad INC, Pittsburgh, PA). The rate of rise was set at $0.2 \mathrm{~s}$ and upper pressure limit was set at $300 \mathrm{psi}$. The standard infusion rate was $3 \mathrm{~mL} / \mathrm{s}$ for the right coronary artery (RCA) and $4 \mathrm{~mL} / \mathrm{s}$ for the left coronary system, but it could be modified in cases of ectasia, anatomic variant or other conditions requiring a customised infusion rate. The contrast volume flushed by the automatic injector for OCT acquisition was calculated with the following formula:

$$
\begin{gathered}
\text { Volume }[\mathrm{mL}]=2 \times \text { infusion rate }[\mathrm{mL} / \mathrm{s}] \times \\
\times \text { imaging time }[\mathrm{s}]
\end{gathered}
$$

The product (infusion rate) $\times$ (imaging time) represents the exact contrast volume required to image a tubular vessel during the exact time required to complete the pullback of SOI. However, 


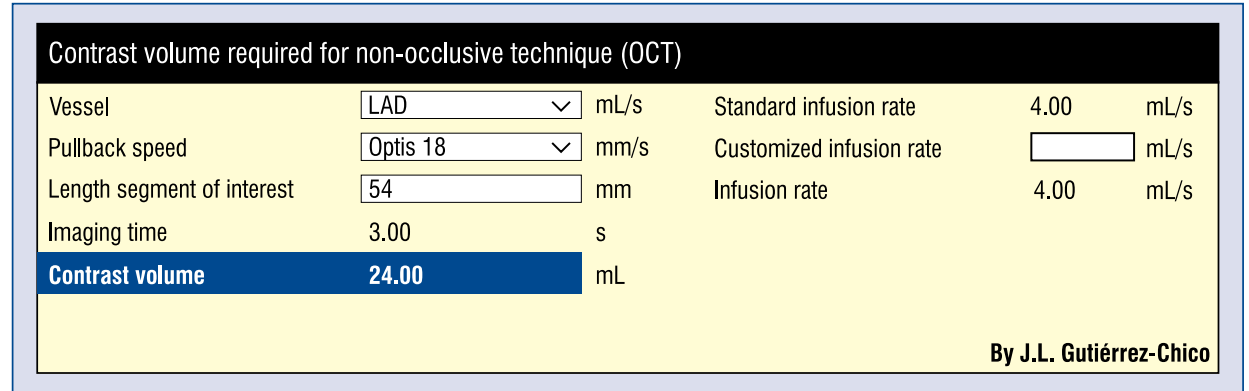

Figure 1. Interface of the online calculator for the contrast volume required in optical coherence tomography with non-occlusive technique. Required parameters: coronary vessel, pullback speed and length of the segment of interest. Optional parameter: customised infusion rate for unusual scenarios; LAD - left anterior descending artery.

a correction factor $(\times 2)$ is empirically required to compensate for contrast volume lost in side branches or non-target vessels, as to adjust for a potential mismatch between contrast arrival and pullback start.

The imaging time is accurately calculated as:

$$
\begin{aligned}
& \text { Imaging time }[\mathrm{s}]=\text { Length of the SOI }[\mathrm{mm}] / \\
& \qquad / \text { pullback speed }[\mathrm{mm} / \mathrm{s}]
\end{aligned}
$$

Combining both equations, the final formula can be summarised as:

Volume $[\mathrm{mL}]=2 \times$ infusion rate $[\mathrm{mL} / \mathrm{s}] \times$ $\times(\mathrm{SOI}$ length $[\mathrm{mm}]) /($ pullback speed $[\mathrm{mm} / \mathrm{s}])$

This formula is implemented in an online calculator, incorporating current pullback speeds and maximal pullback lengths of main OCT manufacturers, so it can be easily calculated in daily routine. https://journals.viamedica.pl/cardiology_journal/ pages/view/calc2 (Fig. 1).

The user-friendly interface requires only three parameters from the user:

- Coronary vessel (to determine the standard infusion rate);

- Pullback speed (mm/s);

- SOI length (mm);

The standard infusion rate can be manually customised in case it is needed.

Alternatively, volume of contrast could be estimated by the operator according to his/her personal experience or following manufacturer recommendations.

\section{OCT analysis}

The SOI was segmented in intervals of $1 \mathrm{~mm}$ and the quality of each cross-section was evaluated by two independent analysts. For the aims of this study, a cross-section was considered of poor quality if the presence of incomplete flushing precluded accurate assessment of a sector $>90^{\circ}$. Otherwise, the section was considered of high quality.

\section{Objectives}

The two main objectives of the study are: quality of OCT acquisition (estimated as the percent of high quality cross-sections per pullback) and amount of contrast used for OCT acquisition. Both variables were compared between a group of pullbacks acquired with accurate contrast volume calculation using the formula and a group of pullbacks acquired with eyeballed volume calculation.

\section{Statistical analysis}

Continuous variables following a Gaussian distribution were reported as mean \pm standard deviation and compared with the t-test for independent samples; if a normal distribution could not be assumed, continuous variables were reported as median (Q1-Q3) and compared with the U-MannWhitney test. Nominal and categorical variables were reported as count (percent) and were compared with the Pearson $\chi^{2}$ (categorical variable) or Fisher exact test (dichotomous variables). In order to adjust the amount of contrast volume for uneven distribution of some variables, a multivariate linear regression model was used, with contrast volume as a dependent variable and stepwise backward selection of the covariates according to likelihood ratio. All statistical analysis was performed using the IBM SPSS 22.0 (IBM Corp, Armonk, NY, USA) software package.

\section{Results}

During the inclusion period 83 patients (160 pullbacks) underwent OCT in the Hospital Frank- 
Table 1. Clinical characteristics of patients.

\begin{tabular}{|c|c|c|c|}
\hline & Eyeball $(n=18)$ & Formula $(n=60)$ & $\mathbf{P}$ \\
\hline Male & $15(83.3)$ & $44(73.3)$ & 0.536 \\
\hline Age & $65.02(9.98)$ & $67.47(16.84)$ & 0.559 \\
\hline Body mass index & $28.17(28.17)$ & $28.57(28.57)$ & 0.778 \\
\hline \multicolumn{4}{|l|}{ CV risk factors } \\
\hline Hypertension & $15(83.3)$ & $52(86.7)$ & 0.709 \\
\hline Hypercholesterolemia & $10(55.6)$ & $25(41.7)$ & 0.418 \\
\hline Diabetes mellitus: & & & 0.391 \\
\hline Type 2 on OAD & $3(16.7)$ & $20(33.3)$ & \\
\hline Type 2 insulin-requiring & $2(11.1)$ & $6(10.0)$ & \\
\hline Smoking: & & & 0.931 \\
\hline Non-smoker & $12(66.7)$ & $42(70.0)$ & \\
\hline Previous smoker & $2(11.1)$ & $5(8.3)$ & \\
\hline Current smoker & $4(22.2)$ & $13(21.7)$ & \\
\hline Family history of CHD & $2(11.1)$ & $0(0.0)$ & 0.051 \\
\hline Previous MI & $9(50.0)$ & $21(35.0)$ & 0.280 \\
\hline \multicolumn{4}{|l|}{ Previous revascularization: } \\
\hline $\mathrm{PCl}$ & $11(61.1)$ & $39(65.0)$ & 0.785 \\
\hline CABG & $0(0.0)$ & $8(13.3)$ & 0.187 \\
\hline GFR (Cockroft-Gault) [mL/min] & 83.18 (39.83) & $89.32(59.96)$ & 0.692 \\
\hline Serum hemoglobin $[\mathrm{g} / \mathrm{dL}]$ & $13.41(1.64)$ & $13.23(1.50)$ & 0.678 \\
\hline LVEF [\%] & $65.6(6.2)$ & $59.8(14.3)$ & 0.025 \\
\hline Clinical indication & & & 0.074 \\
\hline Prognostic indication & $0(0.0)$ & $4(6.7)$ & \\
\hline Stable angina & $13(72.2)$ & 19 (31.7) & \\
\hline Unstable angina & $1(5.6)$ & $5(8.3)$ & \\
\hline NSTEMI & $1(5.6)$ & $12(20.0)$ & \\
\hline STEMI & $0(0.0)$ & $1(1.7)$ & \\
\hline \multicolumn{4}{|l|}{ Procedural variables } \\
\hline LM disease & $2(11.1)$ & $8(13.3)$ & 1.000 \\
\hline LAD disease & $15(83.3)$ & $44(73.3)$ & 0.536 \\
\hline LCx disease & $12(66.7)$ & $43(71.7)$ & 0.770 \\
\hline RCA disease & $11(61.1)$ & $38(63.3)$ & 1.000 \\
\hline Syntax score & $13.28(4.39)$ & $13.96(9.03)$ & 0.671 \\
\hline Contrast volume [mL] & $178(79)$ & $236(114)$ & 0.049 \\
\hline Fluoroscopy time [min] & $18.4(9.2)$ & $21.2(15.3)$ & 0.477 \\
\hline
\end{tabular}

Data presented as counts (\%) or as mean (standard deviation). CABG - coronary artery bypass graft; CHD - coronary heart disease;

CV - cardiovascular; DS - diameter stenosis; GFR - glomerular filtration rate; LAD - left anterior descending; LCx - left circumflex; LM - left main; LVEF - left ventricular ejection fraction; MI - myocardial infarction; NSTEMI - non-ST-segment elevation myocardial infarction; OAD - oral antidiabetics; $\mathrm{PCl}$ - percutaneous coronary intervention; RCA — right coronary artery; SB - side branch;

STEMI - ST-segment elevation myocardial infarction

furt (Oder) in Germany. Five patients (18 pullbacks) were excluded from the analysis because the operator acquired the images with manual injection, 2 pullbacks were excluded because the OCT catheter was occlusive and 3 pullbacks due to NURD. 78 patients (137 pullbacks) were finally analyzed: 60 of them (115 pullbacks) using the formula vs. 18 of them (22 pullbacks) eyeballing the contrast volume. Table 1 presents clinical and angiographic characteristics of the sample. The group using the formula had significantly lower ejection fraction than the eyeball group ( $59.8 \%$ vs. $65.6 \%, \mathrm{p}=0.025)$. The formula group tended to have lower prevalence of family history of coronary 
Table 2. Comparison of optical coherence tomography pullbacks from both groups.

\begin{tabular}{|c|c|c|c|}
\hline & Eyeball $(n=22)$ & Formula $(n=115)$ & $\mathbf{P}$ \\
\hline Pullback speed: & & & 0.0001 \\
\hline $18 \mathrm{~mm} / \mathrm{s}$ & $9(40.9)$ & $95(82.6)$ & \\
\hline $36 \mathrm{~mm} / \mathrm{s}$ & $13(59.1)$ & $20(17.4 \%)$ & \\
\hline SOl length [mm] & $34.68(13.86)$ & $37.10(14.32)$ & 0.462 \\
\hline Right coronary artery & 7 (31.8) & $43(37.4)$ & 0.810 \\
\hline Infusion rate: & & & 0.810 \\
\hline $3 \mathrm{~mL} / \mathrm{s}$ & $7(31.8)$ & $43(37.4)$ & \\
\hline $4 \mathrm{~mL} / \mathrm{s}$ & $15(68.2)$ & $72(62.6)$ & \\
\hline Total number of CS analyzed & 777 & 4252 & \\
\hline Total number of high quality CS & 478 & 4090 & \\
\hline High quality CS [\%] & $63.55(16.41)$ & $96.55(6.27)$ & $<0.0001$ \\
\hline Contrast volume required per pullback $[\mathrm{mL}]$ & $14.55(2.97)$ & $13.03(4.87)$ & 0.057 \\
\hline
\end{tabular}

Data presented as total counts, counts (percent) or as mean (standard deviation). CS — cross-section; SOI — segment of interest

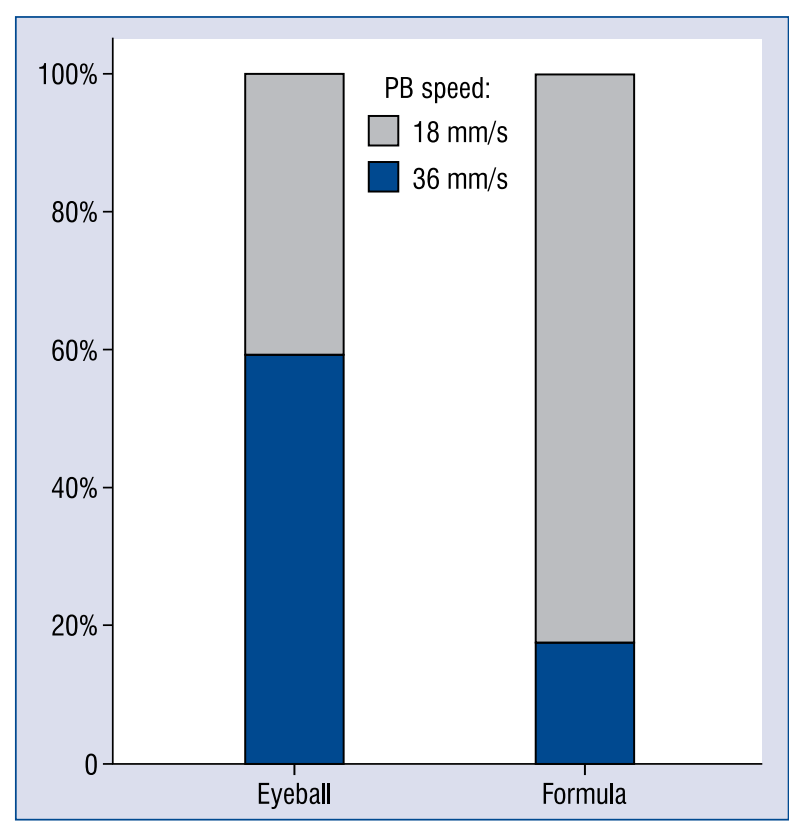

Figure 2. Pullback (PB) speed distribution depending on the method to estimate contrast volume: eyeball estimation vs. calculation with the formula.

artery disease and include more unstable patients with acute coronary syndrome than the eyeball group, but these trends did not reach statistical significance. The total amount of contrast used in interventions of the formula group was significantly higher than in interventions of the eyeball group (236 mL vs. $178 \mathrm{~mL}, \mathrm{p}=0.049$ ).

Table 2 shows the characteristics of OCT pullbacks in both groups. The formula group used

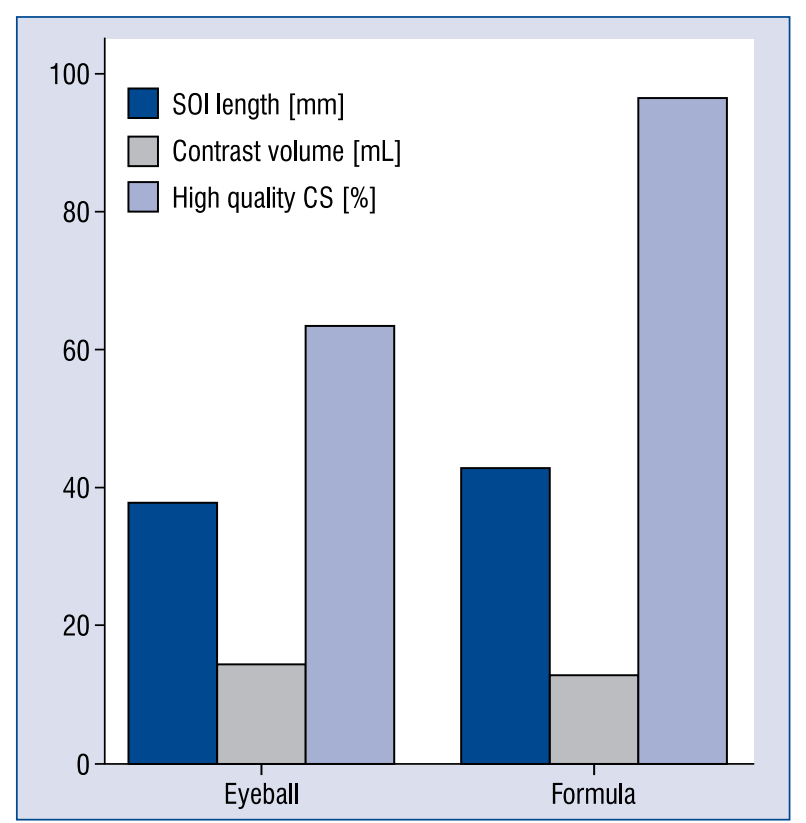

Figure 3. Length of the segment of interest (SOI, $\mathrm{mm}$ ), contrast volume used per pullback $(\mathrm{mL})$ and percent of high quality cross-sections (CS) depending on the method used to estimate contrast volume (eyeball estimation vs. calculation with the formula).

$18 \mathrm{~mm} / \mathrm{s}$ as pullback speed more often than the eyeball group ( $82.6 \%$ vs. $40.9 \%$, p $=0.0001$, Fig. 2 ). There were no significant differences between groups in the length of SOI or in the proportion of pullbacks imaging the RCA (Fig. 3). The average percent of cross-sections with optimal quality per pullback was significantly higher using the formula 
Table 3. Predictors of the contrast volume $[\mathrm{mL}]$ required in multivariate lineal regression analysis.

\begin{tabular}{lccc}
\hline & B & \multicolumn{2}{c}{ 95\% confidence interval } \\
\cline { 3 - 4 } & & Lower limit & Upper limit \\
\hline Segment of interest length $[\mathrm{mm}]$ & 0.27 & 0.24 & 0.31 \\
Pullback speed $(36 \mathrm{mmHg}$ ) & -5.98 & -7.18 & -4.79 \\
Right coronary artery & -3.02 & -3.93 & -2.12 \\
Formula & -4.50 & -5.80 & -3.21 \\
\hline
\end{tabular}

than eyeballing $(96.55 \%$ vs. $63.55 \%, \mathrm{p}<0.0001$, Fig. 3) and interobserver agreement for quality assessment of cross-sections was high: Kappa 0.903 $(\mathrm{p}<0.0001)$.

The formula group tended to use less contrast per OCT pullback than the eyeball group $(13.03 \mathrm{~mL}$ vs. $14.55 \mathrm{~mL}$ ), but this difference did not reach statistical significance $(\mathrm{p}=0.057)$. All factors that might determine the amount of contrast used per OCT pullback were introduced into a multivariate linear regression model, with amount of contrast volume as a dependent variable and the following variables as covariates: pullback speed, SOI length, $\mathrm{RCA}$ and use of the formula for calculation. All variables were significant predictors of the total amount of contrast required per pullback (Table 3 ), with a $\mathrm{R}^{2}=0.704$ of the model. The amount of contrast increased in $0.27 \mathrm{~mL}$ per mm of length of SOI, whilst it decreased in $5.98 \mathrm{~mL}$ per pullback if pullback speed of $36 \mathrm{~mm} / \mathrm{s}$ was used; it also decreased in $3.02 \mathrm{~mL}$ if the RCA was imaged, as compared with arteries of the left system. After correction for all of the above factors, the use of the formula significantly reduced the amount of contrast in $4.50 \mathrm{~mL}$ (Table 3).

\section{Discussion}

The main findings of this study can be summarized in a single statement. The use of a formula to calculate the contrast volume required for OCT acquisition with non-occlusive technique optimises the quality of images whilst reducing the amount of contrast per pullback.

Optical coherence tomography has much higher signal-to-noise ratio than other intracoronary imaging techniques $[1,5]$, which is the key feature enabling fast acquisition of sizable amounts of data in very short time. The shift of all currently available intracoronary OCT systems to Fourier-domain interferometry has increased the acquisition speed even more. Making the most of these characteris- tics, Francesco Prati developed the non-occlusive technique, consisting in acquiring the whole OCT pullback during the time that contrast is infused for a conventional coronary angiography [3, 4]. The time during which the contrast fills the artery and hence removes the blood, is usually enough to grant OCT acquisition. The non-occlusive technique is currently universally accepted and has completely displaced occlusive techniques for clinical coronary applications. Nonetheless, whilst infusion rates for each coronary artery are reasonably standardized, the amount of contrast is usually left to the operator's criterion and is still inconsistently calculated. The education and training on OCT skills are often left to manufacturers and different delegates give generic and inconsistent recommendations that are not always fine-tuned for each specific clinical scenario. For instance, some delegates recommend the infusion of 14-18 $\mathrm{mL}$ for some coronary arteries, irrespective of the length of segment of interest, which is more than required for some targeted applications, whilst it is clearly insufficient for other situations. The amount of contrast is progressively eyeball adjusted by each operator according to personal experience. Furthermore, the use of manual injection is currently promoted by some manufacturers, perhaps to facilitate the adoption of a technique by novel operators, which is a deleterious approach undermining overall quality of images in most of cases.

Calculating the minimal amount of contrast required to warrant maximal quality in OCT images is of utmost importance due to expanding indications of OCT imaging. As a paradigmatic example, the OCTOBER trial (NCT03171311) is currently testing the clinical impact of using OCT to guide some complex bifurcational techniques and therefore it requires several iterations with acquisitions of several OCT pullbacks. An inaccurate calculation of the contrast volume might translate into a substantial increase in the total amount of contrast per procedure and thus jeopardise advantages of a pre- 
cise OCT-guidance. The use of the propozed formula can thereby minimize the amount of contrast required per pullback and still preserve maximal quality, as the present analysis demonstrates. For a comparable length of SOI and for a similar average amount of contrast per pullback, the image quality was significantly higher in the group using the formula than in the group eyeballing the volume. The advantage in sparing contrast by using the formula is not so obvious in unadjusted analysis, because the compared groups have significant differences in the distribution of several variables, mainly in pullback speed: the formula group used preferentially $18 \mathrm{~mm} / \mathrm{s}$, whereas the eyeballing group tended to use more the $36 \mathrm{~mm} / \mathrm{s}$. To adjust the analysis for this uneven distribution, multivariate regression analysis with all the variables that might play a role in determining the amount of contrast was performed, namely: the coronary vessel (RCA, because it determines an infusion rate of $3 \mathrm{~mL} / \mathrm{s}$, vs. any other vessel, imaged with an infusion rate of $4 \mathrm{~mL} / \mathrm{s}$ ), the pullback speed, the length of SOI and use of the formula (vs. eyeballing contrast volume). After adjustment for all these variables, the formula significantly reduced the amount of contrast per pullback (3.21-5.80 $\mathrm{mL}$ on average). The key point for this efficiency is an estimation of the length of SOI, based on angiographic landmarks, and adjustment of the pullback starts at the distal end of SOI. This step is of critical importance to warrant maximal quality exactly in the segment in which it is needed, and it has not been formulated so clearly in any previous study.

In order to avoid confounding factors that might affect the quality of images or the comparability of the groups, or introduce inaccuracies in the data, this was excluded from analysis pullbacks that were acquired with manual infusion and operators in both groups had to define a SOI before acquisition. The average length of SOI was comparable in both groups. By excluding manual injection, potential bias of catheter size was also neutralised: automatic injectors enable a controlled and homogeneous injection of a required amount of contrast at a required infusion rate, adjusting injection pressure to catheter size (e.g. a $4 \mathrm{~F}$ catheter will demand higher injection pressure than a $7 \mathrm{~F}$ catheter to inject the same contrast volume at the same infusion rate). In manual injection, conversely, the operator tends to inject with a relatively stable pressure (limited by his/her manual strength and ergonomics of the syrinx) and this translates into larger contrast volume with large catheter sizes than with small catheter sizes.

\section{Limitations of the study}

This is an observational study, with all the inherent limitations to this kind of design. The distribution of groups was uneven in some variables, particularly in the preferred pullback speed, which required adjusting some results for some potential confounding factors. Moreover, the size of the groups were likewise different, because operators using the formula were probably more methodical and more motivated to use the OCT than the operators in the eyeball groups. Nonetheless, the size of the groups was enough to provide solid statistical results. Furthermore, the current study cannot completely rule out bias due to the operator effect, since the operators in the formula group might be more expert in the use of OCT, as reflected in their use in a more challenging clinical setting (acute coronary syndromes, larger total amount of contrast per intervention, preference for $18 \mathrm{~mm} / \mathrm{s}$ pullback speed which provides higher longitudinal resolution, and other variables). Notwithstanding potential operator effect, the current analysis is enough to validate the formula as a tool to optimize the quality/contrast ratio, because the eventual operator effect would affect only the comparison vs. eyeballing to some extent. Such a comparison under strict scientific conditions of blinding is however very difficult to perform logistically and may have limited clinical interest.

Although it is strongly believed herein, that an automatic injection is critical to guarantee a uniform infusion rate and to optimize the amount of contrast, that very short and proximal segments can be adequately imaged by manually injecting a smaller amount of contrast required cannot be excluded.

\section{Conclusions}

Optical coherence tomography acquisition with non-occlusive technique can be substantially eased by the use of a novel formula to calculate contrast volume required. This method optimizes the quality of images whilst reducing the amount of contrast per pullback.

\section{Conflict of interest: None declared}

\section{References}

1. Gutiérrez-Chico JL, Alegría-Barrero E, Teijeiro-Mestre R, et al. Optical coherence tomography: from research to practice. Eur Heart J Cardiovasc Imaging. 2012; 13(5): 370-384, doi: 10.1093/ /ehjci/jes025, indexed in Pubmed: 22330231. 
2. Windecker S, Kolh P, Alfonso F, et al. 2014 ESC/EACTS Guidelines on myocardial revascularization: The Task Force on Myocardial Revascularization of the European Society of Cardiology (ESC) and the European Association for Cardio-Thoracic Surgery (EACTS)Developed with the special contribution of the European Association of Percutaneous Cardiovascular Interventions (EAPCI). Eur Heart J. 2014; 35(37): 2541-2619, doi: 10.1093/ /eurheartj/ehu278, indexed in Pubmed: 25173339.

3. Prati F, Cera M, Ramazzotti V, et al. Safety and feasibility of a new non-occlusive technique for facilitated intracoronary opti- cal coherence tomography (OCT) acquisition in various clinical and anatomical scenarios. EuroIntervention. 2007; 3(3): 365-370, indexed in Pubmed: 19737719.

4. Prati F, Cera M, Ramazzotti V, et al. From bench to bedside: a novel technique of acquiring OCT images. Circ J. 2008; 72(5): 839-843, indexed in Pubmed: 18441468.

5. Bezerra HG, Costa MA, Guagliumi G, et al. Intracoronary optical coherence tomography: a comprehensive review clinical and research applications. JACC Cardiovasc Interv. 2009; 2(11): 1035-1046, doi: 10.1016/j.jcin.2009.06.019, indexed in Pubmed: 19926041. 